\title{
IceCube as a Neutrino Follow-up Observatory for Astronomical Transients
}

\author{
The IceCube Collaboration ${ }^{\dagger}$ \\ ${ }^{\dagger}$ http://icecube.wisc.edu/collaboration/authors/icrc17_icecube \\ E-mail: kmeaghereulb.ac.be
}

The IceCube Neutrino Observatory is a cubic kilometer neutrino telescope located at the geographic South Pole which can observe neutrinos at energies from $\mathrm{GeV}$ to above a PeV. Now that IceCube has realized its primary goal, the detection of high-energy astrophysical neutrinos, the task remains to identify the sources of these neutrinos. Observing neutrinos in coincidence with transient astrophysical events would be a smoking gun for hadronic acceleration and provide other valuable insights into the nature of the sources. This contribution discusses a new approach to investigate transient phenomena by performing a rapid follow-up analysis. This is done by searching for neutrinos in coincidence with detections by other observatories so that the results can be distributed rapidly to the rest of the astronomical community. In order to accomplish this with minimum delay, the data must be processed immediately at the South Pole and be transmitted to the Northern Hemisphere via the Iridium satellite network. The data processing pipelines, maximum likelihood analysis, and sensitivity of this analysis will be discussed.

Corresponding authors: Kevin Meagher*1

${ }^{1}$ Université Libre de Bruxelles, Science Faculty CP230, B-1050 Brussels, Belgium

35th International Cosmic Ray Conference - ICRC2017

12-20 July, 2017

Bexco, Busan, Korea

${ }^{*}$ Speaker. 


\section{Introduction}

Multimessenger astronomy is the combination of observations of cosmic rays, neutrinos, gravitational waves, and photons of all wavelengths, and it represents a powerful tool to study the nonthermal universe. For quite some time, the IceCube Neutrino Observatory[1] has contributed to multimessenger astronomy by searching for neutrino events correlated with transient astrophysical events observed by other messengers such as gamma-ray bursts[2], active galactic nuclei[3], and gravitational wave events[4]. Despite the fact that IceCube has observed a diffuse flux of astrophysical neutrinos [5], the source of this flux has yet to be identified. Past efforts to correlate IceCube neutrinos with astrophysical transients were hindered by long delays between the original observation and the completion of the IceCube analysis, with results too late to contribute to the observation efforts. This contribution describes a new program to respond to astronomical transients observed by other instruments. These analyses will be performed quickly so that IceCube can issue follow-up notices to the rest of the astronomical community. With this program in place, IceCube will become a full member of the astrophysical community contributing fully to the rapid analysis of astronomical transients. This program is separate from other efforts by the IceCube collaboration to issue circulars generated solely from IceCube data to astronomers[6]. These realtime efforts, such as the Optical, X-ray, and gamma-ray follow-up programs, are documented elsewhere[7, 8, 9].

\section{Selection of Astronomical Transients}

Astronomical transients will be selected from publicly available circulars such as the Astronomer's Telegram (ATEL) ${ }^{1}$ or the Gamma-ray Coordinate Network $(\mathrm{GCN})^{2}$. Analyses may also be initiated upon request by IceCube's MOU partners, and the results will be shared only with the partners according to the rules of the MOU. Transients will be selected based on the following criteria: 1) Potential to generate $\mathrm{TeV}$ neutrinos - the source must exhibit at least one of the following: observation of neutrinos by IceCube or another neutrino telescope, evidence for high energy particle acceleration such as VHE gamma rays or cosmic rays, or theoretical predictions of hadronic acceleration. 2) New or unexpected behavior-The discovery of a new class of astronomical events or unusual behaviors by an otherwise well understood class of events. 3) Brightness-unusually bright or close transients where the probability of neutrino detection is significantly greater than the typical event in its class. 4) General interest - an unusual amount of ATEL or GCN notices for a source of its class. Since this analysis will not be tailored to any specific class of events, it will be capable of being used to investigate any type of sources, particularly new classes which have not been observed yet.

The IceCube Realtime Oversight Committee (ROC) is mandated with oversight and coordinating of IceCube's realtime programs, both those initiated solely with IceCube data such as the optical follow-up [8], gamma-ray follow-up [9] and high-energy starting event (HESE) [10] programs as well as externally triggered analyses such as described in this paper. The members of the ROC were specifically chosen from disparate time zones to ensure that at least one member will be

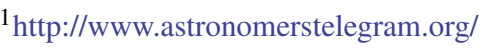

${ }^{2}$ https://gcn.gsfc.nasa.gov/
} 

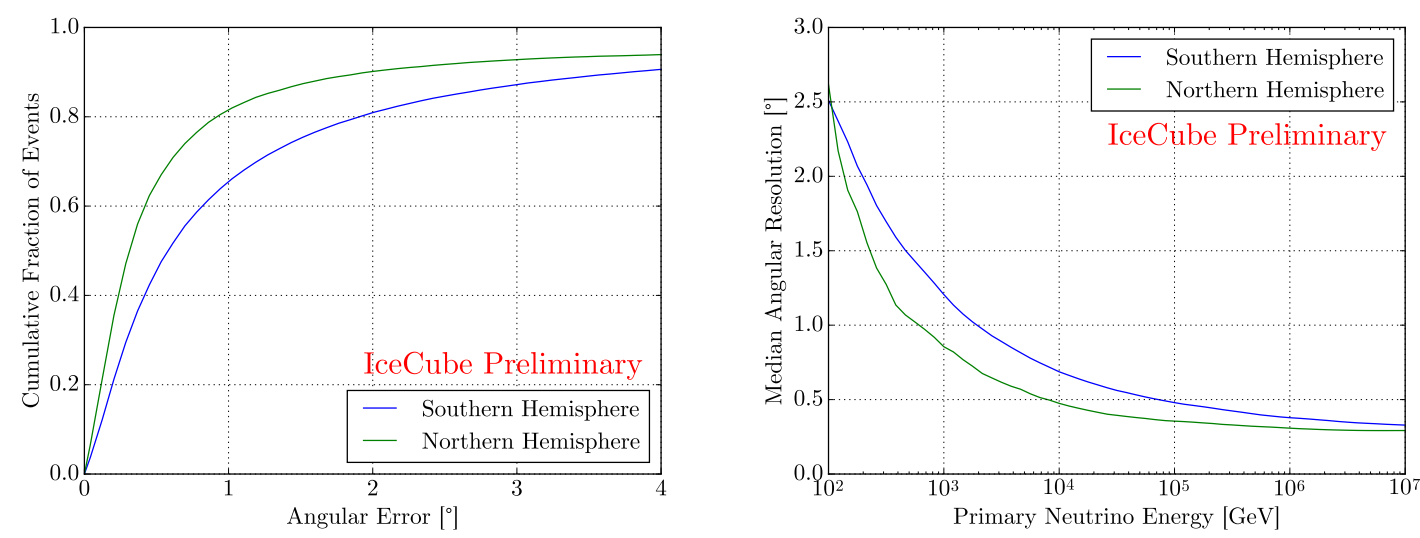

Figure 1: Angular error of events in the online neutrino event selection assuming an $E^{-2}$ energy spectrum. The angular error is the opening angle between the online reconstructed neutrino direction and the true direction in the simulation. The left plot shows the cumulative distribution. The right plot shows the median angular error as a function of the true primary neutrino energy.

active at all times of the day. In order to initiate a realtime analysis the observer will contact the committee with the right ascension, declination, start and stop time, and source extension of the proposed analysis. The approval of at least one ROC member is necessary to initiate the analysis. Upon reception, the ROC will determine whether to issue a circular. In general, for analyses which favor the null-hypothesis, a follow-up circular will not be issued.

\section{Data Processing and Event Selection}

The remote location of IceCube at the Geographical South Pole presents a number of challenges to analyzing data quickly after it is collected. To overcome these obstacles, the IceCube collaboration has developed a realtime data processing pipeline described in detail in references [7, $8,9]$ and summarized here. The IceCube online filtering system contains a computing cluster with $\sim 400 \mathrm{CPU}$ cores which continuously calibrates and reconstructs events as they are recorded by the data acquisition system. This system includes several reconstruction algorithms that characterize each event's extracted light arrival information against the expected patterns from muon neutrino evnets to determine the direction, position and energy of each event [11]. IceCube has many detection channels to reconstruct different types of events based on their flavor and energy. Because of their superior angular resolution $<1^{\circ}$, the muon channel is the most important for multimessenger astronomy. Based on initial reconstructions, approximately $0.2 \%$ of events are selected to be potentially of neutrino origin and are processed with additional, more sophisticated and computationally intensive reconstructions. The selection targets well-reconstructed events with a charge threshold that depends on the reconstructed direction, and with more stringent cuts applied in the atmospheric muon dominated down-going region [9]. The this online event selection has been in operation since 2011, with each year seeing incremental improvements that bring better tools developed offline to the online system.

The results of these reconstructions are used as the input to a multivariate classifier. Further reduction of the atmospheric muon background and separation of an astrophysical signal is achieved 

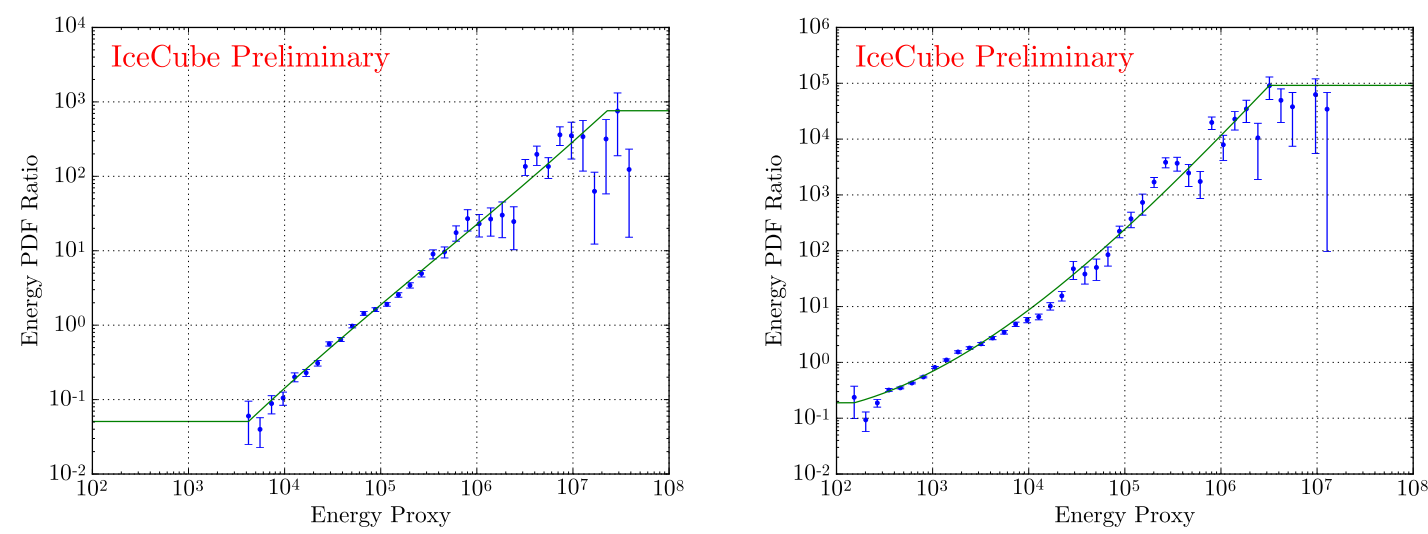

Figure 2: Examples of energy probability distribution function (PDF) ratios. The ratio of the Signal Energy PDF to the Background Energy PDF is shows for two different declinations: $\delta=-15^{\circ}$ (Left) and $\delta=+15^{\circ}$ (Right). The ratio is fit to a $3^{\text {rd }}$ order polynomial (shown in green) for use in the likelihood analysis.

by training boosted decision trees (BDTs) [12]. The training is done separately for the Northern and Southern Hemisphere to account for the different kinds of background encountered in each region, yielding a final event selection rate of $5 \mathrm{mHz}$, equally divided between both hemispheres. A description of the variables entering the BDTs can be found in [9]. Two different subsamples are defined from the output of the BDTs: one for the Northern Hemisphere and a second for the Southern Hemisphere. The neutrino effective area, as used in this analysis, is shown in Fig. 3. The angular error for selected neutrino events, and its dependence on the energy of the neutrino, is shown in Fig. 1. All events selected by the BDT are immediately transferred from the South Pole to the North, where they are made available in a database.

\section{Analysis Method}

This analysis uses an unbinned likelihood method to evaluate if neutrino candidates observed by IceCube are coincident with an astronomical transient. The technique is based on previous work done by the IceCube collaboration for off-line analyses of gamma-ray burst studies [2]. These studies showed that the unbinned likelihood method was more sensitive than simple binned methods. The likelihood of observing $n_{s}$ signal events and $n_{b}$ background events is:

$$
\Lambda\left(\mathbf{x}_{\mathbf{i}} ; n_{s}\right)=-n_{s}+\sum_{i}^{N} \ln \left(\frac{n_{s} \mathrm{~S}\left(\mathbf{x}_{\mathbf{i}}\right)}{\left\langle n_{b}\right\rangle \mathrm{B}\left(\mathbf{x}_{\mathbf{i}}\right)}+1\right)
$$

where $N=n_{s}+n_{b}, \mathrm{~S}(\mathbf{x})$ is the probability density function (PDF) for signal events and $\mathrm{B}(\mathbf{x})$ is the background PDF. $\mathbf{x}_{\mathbf{i}}$ represents the properties of the $i^{\text {th }}$ event. $n_{b}$ can be replaced with $\left\langle n_{b}\right\rangle$, the measured background rate from off-source data to make this a one dimensional likelihood function. For this analysis, the PDFs will include both a spatial and an energy component.

For the spatial signal PDF, the Kent distribution is used. This distribution is the analog of a bivariate normal distribution when projected on to the surface of a unit sphere[13]. The distance from the reconstructed neutrino direction to the source is used as the random variable and the estimated angular uncertainty of the neutrino is used as the width parameter. The error parameter is 

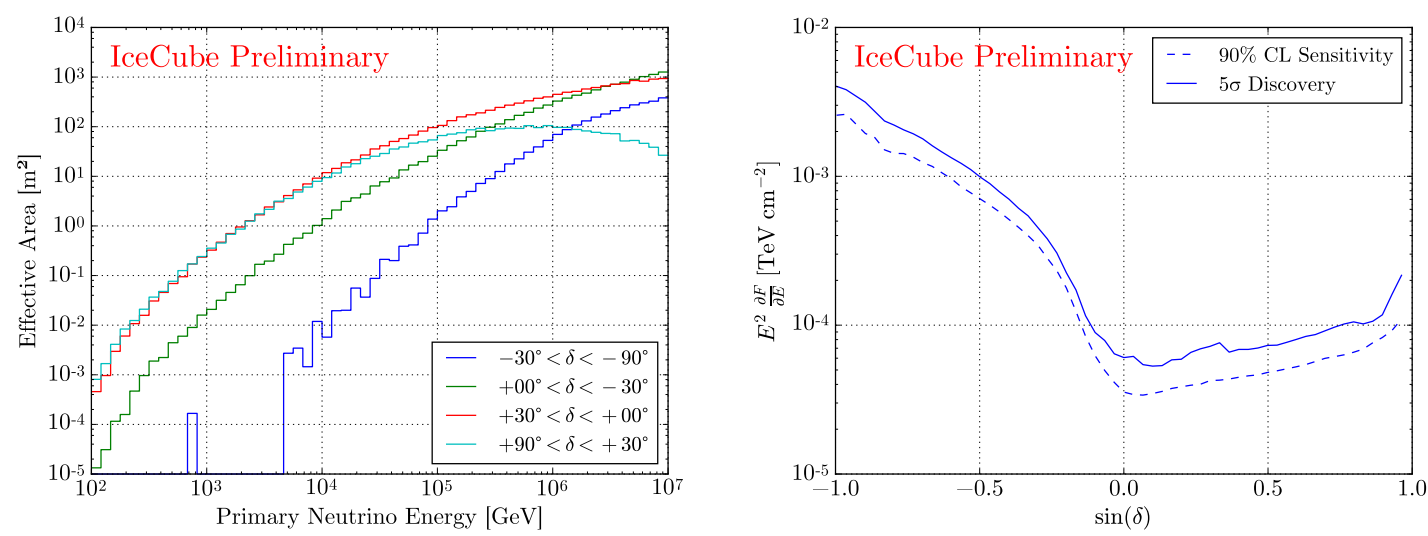

Figure 3: Left Effective areas for the online neutrino event selection. Four different declination bands are shown. At low energies, the Northern Hemisphere has a higher effective area due to the more permissive cuts. However, at high energies, the effective area in the Northern Hemisphere decreases due to absorption in the Earth. Right The performance as a function of declination, using an analysis lasting $10^{5}$ seconds with no source extension. $10^{5}$ seconds is slightly longer than 1 day and is considered the typical timescale for this analyis. Most of the declination dependence is a result of changing background rates. The solid line shows the discovery potential: the minimum flux required to make a $5 \sigma$ discovery $50 \%$ of the time. The dotted line shows the sensitivity: the $90 \%$ confidence upper-limit on the flux at the median value of the test statistic for the background only hypothesis.

the estimated angular uncertainty of the neutrino added in quadrature with the source extension if present. Since the selected events are nearly symmetric in azimuthal directions, a uniform distribution will be used for the spatial background PDF (there is a significant deviation from uniform in the zenith direction but this does not affect the results for an analysis at a single point.)

The shape of the energy PDFs is highly dependent on zenith angle, requiring that the PDF calculation is performed separately for each trigger. For a given trigger, the energy PDFs are constructed empirically from both simulation and off-time data using events from within a $\pm 4^{\circ}$ declination band about the source declination. The signal PDF is calculated using simulated neutrino events weighted to an $E^{-2}$ energy spectrum incident on Earth's surface. Off-time data is used to calculate the background PDF and is augmented with neutrino simulation weighted to an atmospheric spectrum in high-energy bins where there is insufficient statistics from data. The ratio of signal to background is histogrammed and fit to a third order polynomial. In order to avoid unphysical behavior at the highest and lowest energies, the PDF are flattened out above and below the highest and lowest bin respectively. Example energy PDFs are shown in Figure 2. Unlike IceCube GRB analyses, this analysis does not use time PDFs; instead cuts removing data outside the time window of interest are used.

The likelihood analysis provides an estimate of the number of signal events in the data sample $n_{s}$. The analysis will only select events with a signal-to-background ratio greater than 0.1 . For an analysis with a single event, the event needs a signal-to-background ratio of greater than 1 in order to result in a non-zero $n_{s}$. For comparison a typical $100 \mathrm{TeV}$ neutrino within the $1 \sigma$ error circle will have a signal-to-background ratio of 100 and a $1 \mathrm{PeV}$ event could have a ratio as high as $10^{5}$. To calculate the significance of a given analysis, the likelihood value $\Lambda$ is used as a 

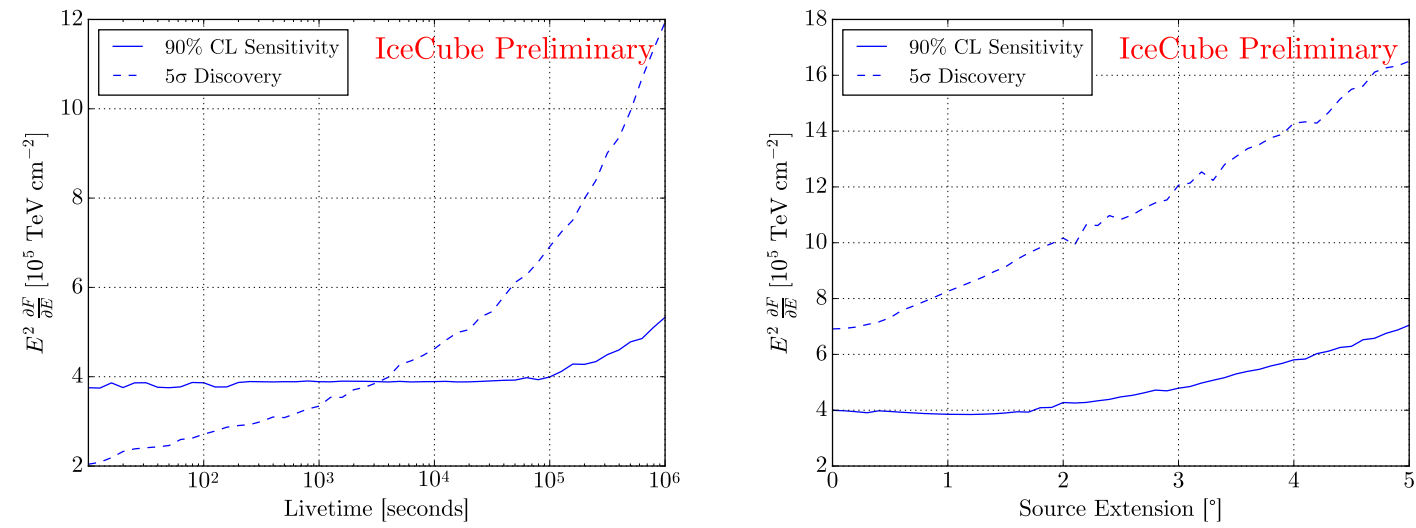

Figure 4: Left: The time dependence of the analysis sensitivity and discovery potential as a function of duration, for a declination of $\delta=+15^{\circ}$ and no source extension. Since the background is low, the sensitivity remains flat for timescales less than $10^{5}$ seconds. Right: Performance as a function of source extension for an analysis with a duration of $10^{5}$ seconds and a declination of $\delta=+15^{\circ}$.

test statistic. Pseudo-experiments with azimuthally scrambled off-time data are used to empirically determine the survival function. Point estimates of the flux or upper-limits are then performed using an iterative search method of pseudo-experiments with both injected off-time data and simulated neutrino events.

To evaluate the performance of the analysis, two figures of merit were defined: the sensitivity and the model discovery potential. The sensitivity is defined as the $90 \%$ confidence upper limit on the median value of the background only test statistic. The discovery potential is defined as the flux in which a $5 \sigma$ discovery will be made $50 \%$ of the time. Plots of these performance metrics are shown in Figures 3 and 4.

\section{Results}

Six transient analyses have been performed to date. This section describes each one briefly.

PS16cgx - On 27 April, 2016 IceCube detected a HESE event with track-like characteristics at location $\alpha=240.57^{\circ}$ and $\delta=+9.34^{\circ}$ with a positional uncertainty of $\Delta \Psi=0.6^{\circ}$ [14]. The Pan-STARRS optical telescope followed up this event three days after the detection and observed 7 supernova candidates consistent with the IceCube event: 6 of them showed flat light curves across 5 days of monitoring consistent with being after peak. However, PS16cgx showed a rising light curve consistent with an explosion at the time of the IceCube event[15]. The trigger time was taken from the IceCube event (05:52:32 UTC) and the position from the Pan-STARRS candidate ( $\left.\alpha=16^{\mathrm{H}} 01^{\mathrm{M}} 18.60^{\mathrm{S}}, \delta=+09^{\circ} 51^{\prime} 53.1^{\prime \prime}\right)$ and a duration of $10^{5}$ seconds (1.16 days) was selected with the trigger time at the center of the time window. The HESE event (RunID $=127853$, EventID $=67093193$ ) was excluded from the likelihood analysis. Only one other event was observed, and it had a large positional uncertainty and a low energy, and therfore $n_{s}=0$.

Cygnus X-3 - In conjunction with a major radio flare, the Fermi-LAT observed an increase in the gamma-ray emission of the microquasar at a significance level of nearly $5 \sigma$ [16]. Using the 


\begin{tabular}{lrrrrr} 
Source & $\begin{array}{r}\text { Start Time } \\
{[\mathrm{UTC}]}\end{array}$ & $\begin{array}{r}\text { Duration } \\
{[\mathrm{D}: \mathrm{H}: \mathrm{M}: \mathrm{S}]}\end{array}$ & RA & Dec & Extension \\
\hline PS16cgx & $2016-04-2615: 59: 12$ & $1: 03: 46: 40$ & $240.33^{\circ}$ & $+09.86^{\circ}$ & $0.0^{\circ}$ \\
Cygnus X-3 & $2017-04-0300: 00: 00$ & $1: 00: 00: 00$ & $308.11^{\circ}$ & $+40.96^{\circ}$ & $0.0^{\circ}$ \\
GRB 170405A & $2017-04-0518: 35: 49$ & $0: 00: 20: 02$ & $219.83^{\circ}$ & $-25.24^{\circ}$ & $0.0^{\circ}$ \\
AGL J0523+0646 & $2017-04-1511: 50: 00$ & $2: 00: 00: 00$ & $080.86^{\circ}$ & $+06.78^{\circ}$ & $0.6^{\circ}$ \\
IceCube 170506A & $2017-05-06$ 00:36:55 & $1: 00: 00: 00$ & $221.80^{\circ}$ & $-26.00^{\circ}$ & $1.0^{\circ}$ \\
AT2017eaw & $2017-05-1012: 00: 00$ & $3: 00: 00: 00$ & $308.68^{\circ}$ & $+60.19^{\circ}$ & $0.0^{\circ}$
\end{tabular}

Table 1: The parameters of each transient anlysis performed to date.

catalog location of Cygnus X-3 microquasar and the time window of the Fermi emission (2017-0403 00:00:00 to 2017-04-04 00:00:00 UTC), a transient analysis was performed. Three events were identified by the unbinned analysis. However, all three were low-energy events with the source outside their $68 \%$ confidence contour, and the final result of the analysis was $n_{s}=0$.

GRB 170405A - Fermi-LAT observed a hard GRB with more than 13 events observed above $100 \mathrm{MeV}$ spatially and temporally correlated with the GRB. The highest-energy photon was a $900 \mathrm{MeV}$ event which is observed 445 seconds after the GBM trigger[17]. Normally, IceCube follow-up analyses are handled by the dedicated gamma-ray burst analyses. But, in this case, due to the interesting nature of this burst, a transient analysis was requested as a cross-check to the GRB analysis. The analysis was performed using the same time windows as the Swift-BAT refined analysis ( $T-239 \mathrm{~s}$ to $T+963 \mathrm{~s}$ where T is the Swift trigger time) [18] and the Enhanced Swift-XRT position $\left(\alpha=14^{\mathrm{H}} 39^{\mathrm{M}} 18.64^{\mathrm{S}}, \delta=-25^{\circ} 14^{\prime} 37.3^{\prime \prime}\right)$ [19]. No events were observed in conjunction with this burst.

AGL J0523+0646 - AGILE reported the appearance of intense gamma-ray emission above $100 \mathrm{MeV}$ located at $\alpha=80.855^{\circ}, \delta=+6.777^{\circ}$ for a period of two days at greater than $6 \sigma$ [20]. Using the same time window as reported by AGILE (2017-04-16 11:50 UT to 2017-04-18 11:50 UT) and the same coordinates ( $\alpha=80.855^{\circ}, \delta=+6.777^{\circ} \pm 0.6^{\circ}$ ) a transient analysis was performed. No events were identified.

IceCube-170506A - on 2017-05-06 12:36:55.80 UT IceCube observed a HESE track-like event[21]. The offline reconstruction yielded a best fit position of $\alpha=221.8^{\circ} \pm 3.0^{\circ}, \delta=-26.0^{\circ}$ $\pm 2.0^{\circ}$, where the error circles represent $90 \%$ containment. To observe any potential clustering of neutrinos a transient analysis was performed, using a time window of \pm 12 hours from the HESE event. The event (RunID $=129474$, EventID $=32674593)$ removed from consideration in the analyis. No further events were observed consistent with the source position.

AT2017eaw - A Supernova candidate was observed on 2017-05-14 located about 2.4' from the center of NGC 6946, a nearby galaxy at $z=0.000133$ and distance $\sim 5.5 \mathrm{Mpc}$ [22]. Although the supernova does not show evidence of strong interactions with the circumstellar medium, the mostly likely scenario for neutrino emission from supernovae, due to its extreme closeness it was deemed interesting enough for a transient analysis. A three day time window was selected from May 10.5 to May 13.5. Four events were observed in coincidence, all of them were low-energy with large positional uncertainty. The likelihood analyis estimated the number of signal events to be $n_{s}=0.742$, with a p-value of $10 \%(1.28 \sigma)$. 


\begin{tabular}{lrrrr} 
Source & $n_{s}$ & p-value & Significance & $\begin{array}{r}90 \% \text { upper limit } \\
{\left[\mathrm{TeV} \mathrm{cm}^{-} 2\right]}\end{array}$ \\
\hline PS16cgx & 0.00 & 1.00 & $0.00 \sigma$ & $3.698 \times 10^{-5}$ \\
CygnusX3 & 0.00 & 1.00 & $0.00 \sigma$ & $5.613 \times 10^{-5}$ \\
GRB 170405A & 0.00 & 1.00 & $0.00 \sigma$ & $5.659 \times 10^{-4}$ \\
AGL J0523+0646 & 0.00 & 1.00 & $0.00 \sigma$ & $4.124 \times 10^{-4}$ \\
IceCube 170506A & 0.00 & 1.00 & $0.00 \sigma$ & $5.482 \times 10^{-5}$ \\
AT2017eaw & 0.74 & 0.10 & $1.28 \sigma$ & $8.871 \times 10^{-5}$
\end{tabular}

Table 2: The results of the likelihood method analysis for each of the transients to date is summarized in this Table. $n_{s}$ is the estimator for the number of signal events.

The parameters of these analyses are summarized in Table 1 and the results are summarized in Table 2. All of the analyses presented here were performed with the 2016-2017 event selection. On May 18, 2017 IceCube began a new data run with an improved BDT for the online event selection. This new selection has improved acceptance to downgoing signal events resulting in a significant improvement in sensitivity in the Southern Hemisphere. In addition, there is an improvement in rejecting poorly-localized background events in both hemispheres, resulting in an improvement in the discovery potential.

\section{References}

[1] IceCube Collaboration, M. G. Aartsen et al., J. Inst. 12 (2017) P03012.

[2] IceCube Collaboration, M. G. Aartsen et al., ApJ 824 (2016) 115.

[3] IceCube Collaboration, M. G. Aartsen et al., ApJ 835 (2017) 45.

[4] ANTARES, IceCube, LIGO Scientific, \& Virgo Collaboration, PRD 93 (2016) 122010.

[5] IceCube Collaboration, M. G. Aartsen et al., ApJ 833 (2016) 3.

[6] IceCube Collaboration, PoS (ICRC2017) 982 (these proceedings).

[7] IceCube Collaboration, M. G. Aartsen et al., Astropart. Phys. 92 (2017) 30.

[8] IceCube Collaboration, M. G. Aartsen et al., $A \& A 539$ (2012) A60.

[9] IceCube, MAGIC, \& VERITAS Collaboration, M. G. Aartsen et al., J. Inst. (2016) P11009.

[10] IceCube Collaboration, M. G. Aartsen et al., PRL 113 (2014) 101101.

[11] IceCube Collaboration, M. G. Aartsen et al., J. Inst. 9 (2014) P03009.

[12] Y. Freund \& R. E. Schapire, J. Comput. Syst. Sci. 55 (1997) 119.

[13] J. T. Kent, J. Royal. Stat. Soc. 44 (1982) 71.

[14] E. Blaufuss, GCN Circ. 19363 (2016).

[15] S. Smartt, GCN Circ. 19381 (2016).

[16] A. Loh \& S. Corbel, ATEL 10243 (2017).

[17] G. Vianello, GCN Circ. 20987 (2017).

[18] A. Lien, GCN Circ. 20999 (2017).

[19] P. Evans et al., GCN Circ. 20985 (2017).

[20] F. Lucarelli, ATEL 10282 (2017).

[21] E. Blaufuss, GCN Circ. 21075 (2017).

[22] S. Dong \& K. Z. Stanek, ATEL 10372 (2017). 